\title{
Forbidding complete hypergraphs as traces
}

\author{
Dhruv Mubayi * \\ Department of Mathematics, Statistics, and Computer Science \\ University of Illinois \\ Chicago, IL 60607 \\ Yi Zhao ${ }^{\dagger}$ \\ Department of Mathematics and Statistics \\ Georgia State University \\ Atlanta, GA 30303
}

September 13, 2006

\begin{abstract}
Let $2 \leq q \leq \min \{p, t-1\}$ be fixed and $n \rightarrow \infty$. Suppose that $\mathcal{F}$ is a $p$-uniform hypergraph on $n$ vertices that contains no complete $q$-uniform hypergraph on $t$ vertices as a trace. We determine the asymptotic maximum size of $\mathcal{F}$ in many cases. For example, when $q=2$ and $p \in\{t, t+1\}$, the maximum is $\left(\frac{n}{t-1}\right)^{t-1}+o\left(n^{t-1}\right)$, and when $p=t=3$, it is $\left\lfloor\frac{(n-1)^{2}}{4}\right\rfloor$ for all $n \geq 3$. Our proofs use the Kruskal-Katona theorem, an extension of the sunflower lemma due to Füredi, and recent results on hypergraph Turán numbers.

\footnotetext{
* Research supported in part by NSF grants DMS-0400812 and an Alfred P. Sloan Research Fellowship. Email: mubayi@math.uic.edu

${ }^{\dagger}$ Research supported in part by NSA grant H98230-06-1-0140. Part of the research conducted while working at University of Illinois at Chicago as a NSF VIGRE postdot. Email: yizhao@mathstat.gsu.edu
} 


\section{Introduction}

Let $[n]=\{1,2, \ldots, n\}$. Given a set $X, 2^{X}$ denotes the family of all subsets of $X$, and $\left(\begin{array}{l}X \\ q\end{array}\right)=\{A \subseteq X:|A|=q\}$. A hypergraph $\mathcal{H}$ on $X$ is a family of subsets of $X$; these subsets are called edges of $\mathcal{H}$ and $X$ is the vertex set of $\mathcal{H}$. If all edges of $\mathcal{H}$ have size $p$, then $\mathcal{H}$ is a $p$-uniform hypergraph ( $p$-graph for short).

Let $G$ be a hypergraph on $X$ and $S \subseteq X$. We define the trace of $G$ on $S$ as

$$
\left.G\right|_{S}:=\{E \cap S: E \in G\} .
$$

Note that we omit multiplicity when defining $G_{S}$.

If there exists a set $S$ such that $\left.G\right|_{S}$ contains a copy of $F$ as a subhypergraph, we say that $G$ contains $F$ as a trace, or $F$ is a trace of $G$. In this case we write $G \rightarrow F$, otherwise $G \nrightarrow F$. Let $L^{p}(n, F)(L(n, F))$ denote the maximum number of edges in a $p$-uniform (not necessarily uniform) hypergraph on $[n]$ not containing $F$ as a trace. Extremal problems on traces started from determining $L\left(n, 2^{[t]}\right)$. Sauer [15], Perles-Shelah [16], and VapnikChervonenkis [18] independently found that $L\left(n, 2^{[t]}\right)=\left(\begin{array}{c}n \\ 0\end{array}\right)+\ldots+\left(\begin{array}{c}n \\ t-1\end{array}\right)$. For the uniform case, Frankl and Pach [6] showed that $L^{p}\left(n, 2^{[t]}\right) \leq\left(\begin{array}{c}n \\ t-1\end{array}\right)$ for $t \leq p \leq n$. Many intersecting problems and applications on traces can be found in the survey of Füredi and Pach [9].

In this paper we consider the problem of forbidding a level of the lattice $2^{[t]}$ as a trace. More precisely, given integers $p, t, n$ with $\max \{p, t\} \leq n$, we study the value of $L^{p}\left(n,\left(\begin{array}{c}{[t]} \\ q\end{array}\right)\right)$ for $1 \leq q \leq t-1$ (the $q=0$ and $q=t$ cases are trivial). Frankl and Pach [6] studied the $q=1$ case and obtained that $\operatorname{ex}\left(p+t-1,\left(\begin{array}{c}{[t]} \\ t-1\end{array}\right)\right) \leq L^{p}\left(n,\left(\begin{array}{c}{[t]} \\ 1\end{array}\right)\right) \leq\left(\begin{array}{c}p+t-1 \\ t-1\end{array}\right)$, where $e x$ is the classical Turán number. Balogh, Keevash and Sudakov [1] investigated the trace problem of forbidding more than one non-trivial level of $2^{[t]}$.

Trivially $L^{p}\left(n,\left(\begin{array}{c}{[t]} \\ q\end{array}\right)\right)=\left(\begin{array}{l}n \\ p\end{array}\right)$ when $p<q$. Therefore throughout the paper we assume that

$$
2 \leq q \leq t-1 \text { and } q \leq p
$$

and whenever we use asymptotic notation, we assume that only $n \rightarrow \infty$. Note that the $p=q$ case is exactly the Turán problem. The reason why we only consider uniform trace numbers is that Füredi and Quinn [10] showed that $L\left(n,\left(\begin{array}{c}{[t]} \\ q\end{array}\right)\right)=L\left(n, 2^{[t]}\right)$ for every $0 \leq q \leq t$, in other words, forbidding a level of the lattice $2^{[t]}$ is equivalent to forbidding the whole lattice in the non-uniform case. Following graph theory language, the forbidden configuration $\left(\begin{array}{c}{[t]} \\ q\end{array}\right)$ is a complete $q$-graph on $t$ vertices, so we denote it by $K_{t}^{q}$, and write $K_{t}=K_{t}^{2}$. 
Our first result, which is little more than an observation, determines the order of magnitude of $L^{p}\left(n, K_{t}^{q}\right)$.

Proposition 1.1. $L^{p}\left(n, K_{t}^{q}\right)=\Theta\left(n^{\min \{p, t-1\}}\right)$.

A trace problem for uniform hypergraphs is in fact a Turán problem. Given a family $\mathcal{F}$ of $r$-graphs, the Turán number $\operatorname{ex}(n, \mathcal{F})$ of $\mathcal{F}$ is the maximum number of edges in an $r$-graph on $n$ vertices containing no $F \in \mathcal{F}$ (see e.g., Füredi [8] for a survey). When $\mathcal{F}=\{F\}$, we write $\operatorname{ex}(n, F)$ instead of $\operatorname{ex}(n,\{F\})$. If we denote $K_{3}$ by $\{12,23,31\}$, then $L^{3}\left(n, K_{3}\right)=\operatorname{ex}\left(n,\left\{F_{1}, F_{2}, F_{3}\right\}\right)$, where $F_{1}=\{124,234,134\}, F_{2}=\{124,234,135\}$, and $F_{3}=$ $\{124,235,136\}$. In general, for any $q$-graph $F$ and $q \leq p$, we have $L^{p}(n, F)=\operatorname{ex}\left(n, \mathcal{H}^{p}(F)\right)$, where $\mathcal{H}^{p}(F)$ is the family of all $p$-graphs $H$ with $|F|$ edges such that $H \rightarrow F$.

Definition 1.2. Let $H_{q, t}^{p}$ be the member of $\mathcal{H}^{p}\left(K_{t}^{q}\right)$ with the maximum number of vertices. In other words, $H_{q, t}^{p}$ is the p-graph obtained from $K_{t}^{q}$ by enlarging each of its $\left(\begin{array}{l}t \\ q\end{array}\right)$ edges with a (different) set of $p-q$ new vertices. Trivially $H_{p, t}^{p}=K_{t}^{p}$.

Since forbidding a family of hypergraphs (as a subgraph) is not easier than forbidding any member of the family,

$$
L^{p}\left(n, K_{t}^{q}\right)=\operatorname{ex}\left(n, \mathcal{H}^{p}\left(K_{t}^{q}\right)\right) \leq \operatorname{ex}\left(n, H_{q, t}^{p}\right) .
$$

Our second result, which is also not hard to prove, shows that the inequality in (2) is asymptotically an equality when $p<t$.

Proposition 1.3. Let $p<t$. Then $L^{p}\left(n, K_{t}^{q}\right)=\operatorname{ex}\left(n, H_{q, t}^{p}\right)+o\left(n^{p}\right)$.

Our main result reduces $L^{p}\left(n, K_{t}^{q}\right)$ when $p \geq t$ to Turán numbers in many cases.

Theorem 1.4. Fix $2 \leq q<t \leq p$. Suppose that $q \in\{t-2, t-1\}$ or $p \in\{t, t+1\}$. Then

$$
L^{p}\left(n, K_{t}^{q}\right)=L^{t-1}\left(n, K_{t}^{q}\right)+o\left(n^{t-1}\right)=\operatorname{ex}\left(n, H_{q, t}^{t-1}\right)+o\left(n^{t-1}\right) .
$$

This suggests that determining $L^{p}\left(n, K_{t}^{q}\right)$ could be as difficult as a hypergraph Turán problem. For example, (3) implies that $L^{4}\left(n, K_{4}^{3}\right)=\operatorname{ex}\left(n, K_{4}^{3}\right)+o\left(n^{3}\right)$, and determining $\operatorname{ex}\left(n, K_{4}^{3}\right)$ is a well-known open problem of Turán [17]. Together with Mantel's Theorem on $\operatorname{ex}\left(n, K_{3}\right)$ [12], Theorem 1.4 gives

$$
L^{p}\left(n, K_{3}\right)=\operatorname{ex}\left(n, K_{3}\right)+o\left(n^{2}\right)=\left(\frac{n}{2}\right)^{2}+o\left(n^{2}\right) .
$$


Determining $\operatorname{ex}\left(n, H_{q, t}^{p}\right)$ in general seems hopeless. However, the $q=2$ case was recently solved by the first author [13] and Pikhurko [14]. Given $2 \leq p \leq \ell$, a $p$-graph is $\ell$-partite if its vertices can be partitioned into $\ell$ classes, such that every edge has at most one vertex from each class. An $\ell$-partite $p$-graph is called complete if it contains all allowable edges. We denote by $T_{\ell}^{p}(n)$ the complete $\ell$-partite $p$-graph (a generalized Turán graph) on $n$ vertices with no two class sizes differ more than one. Let $p<t$. Clearly $T_{t-1}^{p}(n)$ contains no $H_{2, t}^{p}$ as a subgraph and

$$
\left|T_{\ell}^{p}(n)\right|=\sum_{S \in\left(\begin{array}{ll}
{[\ell]} \\
p
\end{array}\right)} \prod_{i \in S}\left\lfloor\frac{n+i-1}{\ell}\right\rfloor=\left(\begin{array}{l}
\ell \\
p
\end{array}\right)\left(\frac{n}{\ell}\right)^{p}+o\left(n^{p}\right) .
$$

The first author [13] showed that $\operatorname{ex}\left(n, H_{2, t}^{p}\right)=\left|T_{t-1}^{p}(n)\right|+o\left(n^{p}\right)$ as $n \rightarrow \infty$. Pikhurko [14] improved this to $\operatorname{ex}\left(n, H_{2, t}^{p}\right)=\left|T_{t-1}^{p}(n)\right|$ for sufficiently large $n$. Applying (2), we thus have $L^{p}\left(n, K_{t}\right) \leq\left|T_{t-1}^{p}(n)\right|$ for sufficiently large $n$. On the other hand, it is easy to see that $T_{t-1}^{p}(n)$ contains no $K_{t}^{q}$ for any $q \geq 2$ as a trace. In fact, every $t$-vertex set $S$ of $T_{t-1}^{p}(n)$ must contain two vertices $a, b$ from the same vertex class, but no edge of $T_{t-1}^{p}(n)$ contains both $a$ and $b$. Thus for $q \geq 2$, every $q$-subset of $S$ containing $a$ and $b$ is absent from $\left.T_{t-1}^{p}(n)\right|_{S}$. Consequently $T_{t-1}^{p}(n) \nrightarrow K_{t}^{q}$, in particular, $L^{p}\left(n, K_{t}\right) \geq\left|T_{t-1}^{p}(n)\right|$. Putting the upper and lower bounds together, for $2 \leq p<t$ and sufficiently large $n$,

$$
L^{p}\left(n, K_{t}\right)=\left|T_{t-1}^{p}(n)\right|=\left(\begin{array}{c}
t-1 \\
p
\end{array}\right)\left(\frac{n}{t-1}\right)^{p}+o\left(n^{p}\right) .
$$

By combining (5) with Theorem 1.4, we obtain the following result.

Corollary 1.5. Suppose that $t=4$ or $p \in\{t, t+1\}$. Then

$$
L^{p}\left(n, K_{t}\right)=\left|T_{t-1}^{t-1}(n)\right|+o\left(n^{t-1}\right)=\left(\frac{n}{t-1}\right)^{t-1}+o\left(n^{t-1}\right) .
$$

We conjecture the values of $L^{p}\left(n, K_{t}^{q}\right)$ as follows.

Conjecture 1.6. Fix $p, q, t, n$ with $2 \leq q<\min \{t, p\}$. For $n>n_{0}$,

$$
L^{p}\left(n, K_{t}^{q}\right)= \begin{cases}\operatorname{ex}\left(n, H_{q, t}^{p}\right) & \text { if } p<t \\ L^{t-1}\left(n-p+t-1, K_{t}^{q}\right)=\operatorname{ex}\left(n-p+t-1, H_{q, t}^{t-1}\right) & \text { if } p \geq t\end{cases}
$$

The equation (5) confirms the conjecture for the case of $q=2, p<t$, and sufficiently large $n$. As further evidence of Conjecture 1.6, we prove its smallest non-trivial case: $(p, q, t)=(3,2,3)$. Note that this sharpens the $p=3$ case of $(4)$. 
Theorem 1.7. Let $n \geq 3$. Then

$$
L^{3}\left(n, K_{3}\right)=\operatorname{ex}\left(n-1, K_{3}\right)=\left\lfloor\frac{(n-1)^{2}}{4}\right\rfloor .
$$

\section{Preliminary Results}

In this section we prove Proposition 1.1, Proposition 1.3 and the supersaturation property for trace problems.

We first observe that $L^{p}\left(n, K_{t}^{q}\right)$ is close to a monotone function of $n$.

Proposition 2.1. $L^{p}\left(n, K_{t}^{q}\right) \geq L^{p-i}\left(n-i, K_{t}^{q}\right)$ for $1 \leq i \leq p-q$.

Proof. Suppose that $G \subseteq\left(\begin{array}{c}{[n-i]} \\ p-i\end{array}\right)$ satisfies $G \nrightarrow K_{t}^{q}$. We extend $G$ to a $p$-graph $G^{\prime}$ by adding a set $C$ of $i$ new vertices and replacing each $E \in G$ by $E \cup C$. We claim that $G^{\prime} \nrightarrow K_{t}^{q}$. Consider a $t$-set $S$ of vertices. If $S$ contains a vertex $x \in C$, then all edges of $G^{\prime}$ contain $x$, and consequently all $q$-subsets of $E \backslash\{x\}$ are absent from $\left.G^{\prime}\right|_{E}$. Otherwise $E \cap C=\emptyset$, and we have $\left.G^{\prime}\right|_{E}=\left.G\right|_{E} \nrightarrow K_{t}^{q}$.

Proof of Proposition 1.1. We need to show that $L^{p}\left(n, K_{t}^{q}\right)=\Theta\left(n^{\min \{p, t-1\}}\right)$. When $p \geq$ $t$, Frankl and Pach [6] showed that $L^{p}\left(n, K_{t}^{q}\right) \leq\left(\begin{array}{c}n \\ t-1\end{array}\right)$. When $p<t$, trivially $L^{p}\left(n, K_{t}^{q}\right) \leq$ $\left(\begin{array}{l}n \\ p\end{array}\right)$. We now consider lower bounds. When $p \leq t-1$, Since $T_{t-1}^{p}(n) \nrightarrow K_{t}^{q}$ (for $q \geq 2$ ), we have $L^{p}\left(n, K_{t}^{q}\right) \geq\left|T_{t-1}^{p}(n)\right|=\Omega\left(n^{p}\right)$. Now let $p \geq t$. Since $T_{t-1}^{t-1}(n-p+t-1) \nrightarrow K_{t}^{q}$ and $\left|T_{t-1}^{t-1}(n-p+t-1)\right|=\Omega\left(n^{t-1}\right)$, we have $L^{t-1}\left(n-p+t-1, K_{t}^{q}\right)=\Omega\left(n^{t-1}\right)$. Proposition 2.1 thus implies that $L^{p}\left(n, K_{t}^{q}\right) \geq L^{t-1}\left(n-p+t-1, K_{t}^{q}\right)=\Omega\left(n^{t-1}\right)$.

Definition 2.2. Let $F$ be a p-graph $(p \geq 2)$ on $[\ell]$ and $\vec{m}=\left\langle m_{1}, \ldots, m_{\ell}\right\rangle$ be a vector of positive integers. The blow-up $F(\vec{m})$ of $F$ is obtained by replacing each vertex $i$ by a vertex class $V_{i}$ of size $m_{i}$, and each edge $\left\{i_{1}, \ldots, i_{p}\right\}$ by the family of all p-sets $\left\{w_{1}, \ldots, w_{p}\right\}$, where $w_{j} \in V_{i_{j}}$. We simply write $F(m)$ if all $m_{i}=m$.

A phenomenon discovered by Brown, Erdős and Simonovits [5], usually called supersaturation, implies that $\operatorname{ex}(n, F(\vec{m}))=\operatorname{ex}(n, F)+o\left(n^{r}\right)$ for every $r$-graph $F$ and its blow-up $F(\vec{m})$. To prove Proposition 1.3, we need a lemma from [13], which is a simple consequence of supersaturation. 
Lemma 2.3 (Lemma 4 in [13]). Let $m, p$ be positive integers with $p \geq 2$, and let $\mathcal{F}$ be a finite family of p-graphs. If $H$ is a p-graph satisfying $H \subseteq F(m)$ for all $F \in \mathcal{F}$, then $\operatorname{ex}(n, H) \leq \operatorname{ex}(n, \mathcal{F})+o\left(n^{p}\right)$.

Proof of Proposition 1.3. Here $p<t$ and we must show that $L^{p}\left(n, K_{t}^{q}\right)=\operatorname{ex}\left(n, H_{q, t}^{p}\right)+$ $o\left(n^{p}\right)$. Because of $(2)$, we only need to show that $L^{p}\left(n, K_{t}^{q}\right) \geq \operatorname{ex}\left(n, H_{q, t}^{p}\right)+o\left(n^{p}\right)$ when $n \rightarrow \infty$. For each $\left.F \in \mathcal{H}^{p}\left(K_{t}^{q}\right)\right)$, it is easy to see that $H_{q, t}^{p} \subseteq F\left(\left(\begin{array}{l}t \\ q\end{array}\right)\right)$. Lemma 2.3 implies that

$$
\operatorname{ex}\left(n, H_{q, t}^{p}\right) \leq \operatorname{ex}\left(n, \mathcal{H}^{p}\left(K_{t}^{q}\right)\right)+o\left(n^{p}\right)=L^{p}\left(n, K_{t}^{q}\right)+o\left(n^{p}\right)
$$

Next we prove the supersaturation phenomenon for trace problems.

Lemma 2.4. $L^{p}\left(n, K_{t}^{q}(m)\right) \leq L^{p}\left(n, K_{t}^{q}\right)+o\left(n^{p}\right)$. In particular, $L^{p}\left(n, K_{t}^{q}(m)\right)=(1+$ $o(1)) L^{p}\left(n, K_{t}^{q}\right)$ for $p<t$.

Proof. The second assertion follows from the first by realizing that $L^{p}\left(n, K_{t}^{q}\right)=\Theta\left(n^{p}\right)$ for $p<t$ from Proposition 1.1. To prove the first claim, recall that $\mathcal{H}^{p}\left(K_{t}^{q}(m)\right)$ is the family of $p$-graphs whose $\left|K_{t}^{q}(m)\right|$ edges contain $K_{t}^{q}(m)$ as a trace, and $H_{q, t}^{p}$ is obtained from $K_{t}^{q}$ by enlarging each of its $\left(\begin{array}{l}t \\ q\end{array}\right)$ edges with a different set of $p-q$ new vertices. Let $\tilde{H}=H_{q, t}^{p}(\vec{m})$, where $m_{i}=m$ for all the vertices $v_{i}$ in the original $K_{t}^{q}$, and $m_{i}=1$ for the other vertices. It is easy to see that $\tilde{H}$ is a member of $\mathcal{H}^{p}\left(K_{t}^{q}(m)\right)$. We thus have

$$
\begin{aligned}
L^{p}\left(n, K_{t}^{q}(m)\right) & =\operatorname{ex}\left(n, \mathcal{H}^{p}\left(K_{t}^{q}(m)\right)\right) \\
& \leq \operatorname{ex}(n, \tilde{H}) \\
& \leq \operatorname{ex}\left(n, H_{q, t}^{p}\right)+o\left(n^{p}\right) \\
& \leq L^{p}\left(n, K_{t}^{q}\right)+o\left(n^{p}\right),
\end{aligned}
$$

where the first inequality holds because $\tilde{H} \in \mathcal{H}^{p}\left(K_{t}^{q}(m)\right)$, the second inequality holds because of supersaturation for the Turán problems, and the last one holds because of (7).

\section{Proof of Theorem 1.4}

Throughout this section we will assume that $p \geq t$. Our goal is to prove that if $q \in$ $\{t-2, t-1\}$ or $p \in\{t, t+1\}$, then

$$
L^{p}\left(n, K_{t}^{q}\right)=L^{t-1}\left(n, K_{t}^{q}\right)+o\left(n^{t-1}\right) .
$$


In fact, the second equality of (3) in Theorem 1.4, $L^{t-1}\left(n, K_{t}^{q}\right)=\operatorname{ex}\left(n, H_{q, t}^{t-1}\right)+o\left(n^{t-1}\right)$, follows from Proposition 1.3 (note that the second condition in (1) still holds because $t-1 \geq q)$. Furthermore, we claim that

$$
L^{p}\left(n, K_{t}^{q}\right) \geq L^{t-1}\left(n, K_{t}^{q}\right)+o\left(n^{t-1}\right) .
$$

To see this, first observe that Proposition 2.1 implies that $L^{p}\left(n, K_{t}^{q}\right) \geq L^{t-1}\left(n-p+t-1, K_{t}^{q}\right)$. Proposition 1.3 further gives that $L^{p}\left(n, K_{t}^{q}\right) \geq \operatorname{ex}\left(n-p+t-1, H_{q, t}^{t-1}\right)+o\left(n^{t-1}\right)$. Now we recall a fact on the Turán number, which immediately follows from the existence of $\lim _{n \rightarrow \infty} \operatorname{ex}(n, \mathcal{F}) /\left(\begin{array}{l}n \\ p\end{array}\right)$. Given a family $\mathcal{F}$ of $r$-graphs and an integer $c>0$,

$$
\operatorname{ex}(n, \mathcal{F})-\operatorname{ex}(n-c, \mathcal{F})=o\left(n^{r}\right) .
$$

Therefore $L^{p}\left(n, K_{t}^{q}\right) \geq \operatorname{ex}\left(n, H_{q, t}^{t-1}\right)+o\left(n^{t-1}\right)$ and (8) follows after applying Proposition 1.3 again.

Therefore the main task is to verify

$$
L^{p}\left(n, K_{t}^{q}\right) \leq L^{t-1}\left(n, K_{t}^{q}\right)+o\left(n^{t-1}\right) .
$$

for $q \in\{t-2, t-1\}$ or $p \in\{t, t+1\}$. The $q=t-1$ case (Section 3.1) is the easiest: its main idea is to find a one-to-one function from a $p$-graph $G$ with $G \nrightarrow K_{t}^{q}$ to a $(t-1)$-graph $G^{\prime}$ such that $G^{\prime} \nsupseteq K_{t}^{t-1}$. The remaining cases are harder: we present two lemmas in Section 3.2, and complete the proofs in Section 3.3. The main tools include the Erdös-Ko-Rado theorem, the Kruskal-Katona theorem and a lemma on sunflowers due to Füredi.

\section{1. $q=t-1$.}

Let $G$ be a hypergraph and $S$ be a subset of its vertex set. The degree of $S$ in $G, \operatorname{deg}_{G}(S)$, or $\operatorname{deg}(S)$ if the underlying hypergraph is clear from the context, is the number of edges in $G$ containing $S$ (frequently called codegree when $|S| \geq 2$ ). Given a $p$-graph $G$, if every edge $E \in G$ contains at least one $p^{\prime}$-subset $E^{\prime}$ with $\operatorname{deg}_{G}\left(E^{\prime}\right)=1$, then $\phi(E)=E^{\prime}$ defines a one-to-one function from $G$ to $G^{\prime}=\left\{E^{\prime}: E \in G\right\}$ (if more than one $p^{\prime}$-subsets are of degree 1 , then arbitrarily pick one of them to be $\phi(E))$.

Proposition 3.1. Let $G$ be a p-graph such that $G \nrightarrow K_{t}^{q}$. If there exists a function $\phi$ mapping every edge $E \in G$ to a q-set $E^{\prime} \subset E$ such that $\operatorname{deg}_{G}\left(E^{\prime}\right)=1$, then $\phi(G)=\{\phi(E)$ : $E \in G\}$ contains no $K_{t}^{q}$ as a subgraph. 
Proof. Suppose instead, that $G^{\prime}$ contains a subgraph $G_{1}^{\prime}$ on a $t$-set $T$ such that $G_{1} \cong K_{t}^{q}$. Clearly $\phi$ is one-to-one. Let $\phi^{-1}$ be the inverse function. We claim that each edge $E \in G$ with $\phi(E) \in G_{1}^{\prime}$ satisfies that $E \cap T=\phi(E)$ and therefore $\left.G\right|_{T} \supseteq K_{t}^{q}$, contradicting the assumption that $G \nrightarrow K_{t}^{q}$. In fact, if $E \cap T \supsetneq \phi(E)$, then $E \cap T$ contains another $q$-set $Q \in G_{1}^{\prime}$. Clearly $E \neq \phi^{-1}(Q)$ because $\phi$ is a function. The fact that both $E$ and $\phi^{-1}(Q)$ contain $Q$ implies that $\operatorname{deg}_{G}(Q) \geq 2$, a contradiction.

The following lemma is the key observation for proving the $q=t-1$ case of (10).

Lemma 3.2. Let $2 \leq t \leq p$. Suppose that $S$ is a p-set and $H$ is a family of proper subsets of $S$. If every $(t-1)$-subset of $S$ is contained in some member of $H$, then $H \rightarrow K_{t}^{t-1}$.

Proof. We do induction on $p$ for fixed $t \geq 2$. The base case $p=t$ is trivial, since every $(t-1)$-subset of $S$ is a member of $H$, or $\left(\begin{array}{c}S \\ p-1\end{array}\right) \subseteq H$. For the induction step, let $p>t$ and consider two cases. If $\left(\begin{array}{c}S \\ p-1\end{array}\right) \subseteq H$, then for a fixed $t$-set $T \subset S$, we have $\left.H\right|_{T} \supseteq\left(\begin{array}{c}T \\ t-1\end{array}\right)$ because each $(t-1)$-subset $T^{\prime}$ of $T$ is contained in $T^{\prime} \cup(S \backslash T)$, which is a member of $H$. Otherwise $\left(\begin{array}{c}S \\ p-1\end{array}\right) \nsubseteq H$, and there exists an $(p-1)$-set $S^{\prime} \notin H$. It is easy to see that $\left.H\right|_{S^{\prime}}$ satisfies the assumption of the lemma with $p-1$ instead of $p$. We then apply the induction hypothesis to $S^{\prime}$ and $\left.H\right|_{S^{\prime}}$ obtaining that $\left.H\right|_{S^{\prime}} \rightarrow K_{t}^{t-1}$ and consequently $H \rightarrow K_{t}^{t-1}$.

Proof of (10) for $q=t-1$. Let $G$ be an $n$-vertex $p$-graph not having $K_{t}^{t-1}$ as a trace. Each edge $E \in G$ must contain a $(t-1)$-subset $E^{\prime}$ with $\operatorname{deg}_{G}\left(E^{\prime}\right)=1$, otherwise we apply Lemma 3.2 with $S=E$ and $H=\left.G\right|_{E}-\{E\}$ to conclude that $G \rightarrow K_{t}^{t-1}$. We thus define $\phi(E)=E^{\prime}$ and $\phi$ is a one-to-one function from $G$ to $\left(\begin{array}{c}{[n]} \\ t-1\end{array}\right)$. By Proposition 3.1, the resulting $(t-1)$-graph $G^{\prime}$ contains no $K_{t}^{t-1}$ as a subgraph, thus $|G|=\left|G^{\prime}\right| \leq \operatorname{ex}\left(n, K_{t}^{t-1}\right)=$ $L^{t-1}\left(n, K_{t}^{t-1}\right)$.

\subsection{Two Lemmas}

Fix $G \subseteq\left(\begin{array}{c}{[n]} \\ p\end{array}\right)$ with $|G| \geq 2$. The following partition of $G$ will be needed in our proofs. Define a function $f: G \rightarrow[p]$ such that for $E \in G$,

$$
f(E)=\min \{|D|: D \subseteq E, \operatorname{deg}(D)=1 \text { and } \forall S \subset D, \operatorname{deg}(S) \geq 2\} .
$$

(Throughout this subsection $\operatorname{deg}=\operatorname{deg}_{G}$.) Since $\operatorname{deg}(E)=1$ and $\operatorname{deg}(\emptyset)=|G| \geq 2$, there always exists a subset $D \subset E$ such that $\operatorname{deg}(D)=1$ but $\operatorname{deg}(S) \geq 2$ for all $S \subset D$. Hence $f$ 
is well defined. For $1 \leq i \leq p$, let $G_{i}=\{E \in G: f(E)=i\}$. Clearly, $G_{p}+G_{p-1}+\ldots+G_{1}$ is a partition of $G$.

Furthermore, for $k \leq p$, let $\partial^{k} G$ denote the shadow of $G$ at level $k$, namely, $\partial^{k} G=\{D$ : $|D|=k, D \subseteq E$ for some $E \in G\}$. In particular, $\partial G=\partial^{p-1} G$. Let

$$
G^{i}=\left\{D \in \partial^{i} G: \operatorname{deg}(D)=1 \text { and } \forall S \subset D, \operatorname{deg}(S) \geq 2\right\} .
$$

If we map each $D \in G^{i}$ to the unique $E \in G$ such that $D \subseteq E$, then we obtain an onto function from $G^{i}$ to $G_{i}$. Hence $\left|G_{i}\right| \leq\left|G^{i}\right|$ for $1 \leq i \leq p$. We are ready to state two lemmas, which are the key ingredients in our proofs.

Lemma 3.3. Let $t \leq k \leq p$ and $G \subseteq\left(\begin{array}{c}{[n]} \\ p\end{array}\right)$. If $G \nrightarrow K_{t}^{q}$, then $\left|\partial^{t}\left(G^{k}\right)\right|=O\left(n^{t-2}\right)$.

Lemma 3.4. Let $t \leq i \leq k \leq p$ and $G \subseteq\left(\begin{array}{c}{[n]} \\ p\end{array}\right)$. If $G \nrightarrow K_{t}^{t-2}$, then $\left|\partial^{i}\left(G^{k}\right)\right|=O\left(n^{t-2}\right)$.

In order to prove Lemma 3.3. We need the following lemma on sunflowers, which is an easy corollary of a result of Füredi [7] and the Erdös-Ko-Rado Theorem [4]. A sunflower (or $\Delta$-system) with $k$ petals and a core $Y$ is a collection of distinct sets $S_{1}, \ldots, S_{k}$ such that $S_{i} \cap S_{j}=Y$ for all $i \neq j$.

Lemma 3.5. Given $k$ and $r$, there exists $C=C(k, r)$ such that every $F \subseteq\left(\begin{array}{c}{[n]} \\ k\end{array}\right)$ with $|F| \geq C n^{k-i}$ contains an $r$-petal sunflower with a core of size less than $i$.

Proof. Füredi [7] extended the well-known Sunflower Lemma of Erdős and Rado [3] as follows: given $k$ and $r$, there exists $c=c(k, r)$ such that every $F \subseteq\left(\begin{array}{c}{[n]} \\ k\end{array}\right)$ contains a subfamily $F^{\prime}$ such that $\left|F^{\prime}\right|>c|F|$ and for all distinct $E_{1}, E_{2} \in F^{\prime}, F^{\prime}$ contains an r-petal sunflower with core $E_{1} \cap E_{2}$. (The original statement in [7] is actually stronger.) Let $C=1 / c$. We apply this result to $F \subseteq\left(\begin{array}{c}{[n]} \\ k\end{array}\right)$ with $|F| \geq n^{k-i} / c$. Since $\left|F^{\prime}\right| \geq n^{k-i}>\left(\begin{array}{c}n-i \\ k-i\end{array}\right)$, by the ErdősKo-Rado Theorem [4], $F^{\prime}$ contains $E_{1}, E_{2}$ such that $\left|E_{1} \cap E_{2}\right|<i$. Then $F^{\prime}$ contains an $r$-petal sunflower with core $E_{1} \cap E_{2}$ of size less than $i$.

Fix $i \in[p]$. We say that a hypergraph $H \subseteq \partial^{i} G$ satisfies the property $(\diamond)$ if

$$
\text { for all } D \in H \text { and } x \in D \text {, there exists } E \in G \text { s.t. } D \backslash\{x\} \subset E, x \notin E \text {. }
$$

We claim that $\partial^{i} G^{k}$ satisfies $(\diamond)$ for all $t \leq i \leq k$. First we show that $G^{k}$ satisfies $(\diamond)$. Pick $D \in G^{k}$ and $x \in D$. Since $D \in G^{k}$, there exists a unique $E_{1} \in G$ such that $D \subseteq E_{1}$. Since $\operatorname{deg}(D \backslash\{x\}) \geq 2$, there exists $E \in G, E \neq E_{1}$ such that $D \backslash\{x\} \subset E$. In addition, $x \notin E$, 
otherwise $D \subseteq E$, contradicting $\operatorname{deg}(D)=1$. We next observe that if $H$ satisfies $(\diamond)$, then $\partial H$ also satisfies $(\diamond)$. In fact, let $S \in \partial H$ and $x \in S$. Suppose that $S \subset D \in H$. Then there exists $E \in G$ such that $D \backslash\{x\} \subset E, x \notin E$, in particular, $S \backslash\{x\} \subset E$.

Given a function $\phi: A \rightarrow B$ and $y \in B$, let $\phi^{-1}(y)=\{x \in A: \phi(x)=y\}$.

Proof of Lemma 3.3. Let $H=\partial^{t}\left(G^{k}\right)$. Since $G \nrightarrow K_{t}^{q}$, each $D \in H$ contains at least one $q$-element subset $Q$ such that $Q \notin G_{D}$. We denote such a $Q$ by $\psi(D)$ (arbitrarily pick one if more than one set can be chosen). In order to show that $|H|=O\left(n^{t-2}\right)$, it suffices to show that for each set $Q \in\left(\begin{array}{c}{[n]} \\ q\end{array}\right)$, we have $\left|\psi^{-1}(Q)\right|=O\left(n^{t-2-q}\right)$. Define a $(t-q)$-graph $F=\left\{D-Q: D \in \psi^{-1}(Q)\right\}$. Suppose to the contrary, that $\left|\psi^{-1}(Q)\right|=|F|>C n^{t-q-2}$ for the constant $C=C(t-q, p-t+3)$ from Lemma 3.5. By Lemma 3.5, $F$ contains a sunflower $S_{1}, \ldots, S_{p-t+3}$ with core $Y$ of size at most 1 . For all $i$, let $D_{i}=S_{i} \cup Q \in H$.

Case 1: $Y=\emptyset$. Since $D_{1} \in \partial^{t}\left(G^{k}\right)$, there exists $E \in G$ such that $D_{1} \subset E$. At most $\left|E \backslash D_{1}\right|=p-t$ petals have non-empty intersection with $E \backslash D_{1}$. Since the total number of petals is greater than $p-t+1$, there exists $j \neq 1$ such that $S_{j} \cap\left(E \backslash D_{1}\right)=\emptyset$, or $D_{j} \cap E=Q$, a contradiction.

Case 2: $Y=\{x\}$. Since $H$ satisfies $(\diamond)$, there exists $E \in G$ such that $D_{1} \backslash\{x\} \subset E$ and $x \notin E$. At most $\left|E \backslash\left(D_{1} \backslash\{x\}\right)\right|=p-t+1$ petals have non-empty intersection with $E \backslash\left(D_{1} \backslash\{x\}\right)$. Since the total number of petals is $p-t+3$, there exists $j, j \neq 1$ such that $S_{j} \cap\left(E \backslash\left(D_{1} \backslash\{x\}\right)\right)=\emptyset$. Since $x \notin E$ but $x \in D_{j}$, we have $D_{j} \cap E=Q$, a contradiction.

Proof of Lemma 3.4. We do induction on $i \geq t$. The base case $i=t$ holds because of Lemma 3.3. Let $H=\partial^{i} G^{k}$. For each $D \in H$, arbitrarily pick one of its $t$-subsets $S$. Since $G \nrightarrow K_{t}^{t-2}, S$ contains a $(t-2)$-subset $Q$ such that $\left.Q \notin G\right|_{S}$. Suppose $S \backslash Q=\{x, y\}$. Let $\psi(D)=D-\{y\}$ and $\phi(D)=(\psi(D), Q, x)$. We claim that $\left|\psi^{-1}(D-\{y\})\right| \leq\left(\begin{array}{c}i-1 \\ t-1\end{array}\right)(t-1)(p-$ $i+2)$. By the pigeonhole principle, it suffices to show that $\left.\mid \phi^{-1}(D-\{y\}, Q, x)\right) \mid \leq p-i+2$ (for a fixed $D-\{y\}$, there are $\left(\begin{array}{c}i-1 \\ t-1\end{array}\right)(t-1)$ ways of choosing a $(t-2)$-set $Q$ and an element $x \notin Q)$. Suppose instead, that there exist $D_{1}, \ldots, D_{p-i+3} \in H$ forming a sunflower with core $D-\{y\}$ and petals $\left\{y_{j}\right\}, 1 \leq j \leq p-i+3$ such that $\left.Q \notin G\right|_{S_{j}}$ for $S_{j}=Q \cup\left\{x, y_{j}\right\}$. Since $H$ satisfies $(\diamond)$, there exists $E \in G$ such that $D_{1} \backslash\{x\} \subset E$ and $x \notin E$. At most $\left|E \backslash\left(D_{1} \backslash\{x\}\right)\right|=p-i+1$ petals have non-empty intersection with $E \backslash\left(D_{1} \backslash\{x\}\right)$. Since the total number of petals is $p-t+3$, there exists $j \neq 1$ such that $y_{j} \notin E$. Since $x \notin E$ but $x \in D_{j}$, we have $S_{j} \cap E=Q$, a contradiction.

We thus have $|H| \leq C|\psi(H)|$, where $C=\left(\begin{array}{c}i-1 \\ t-1\end{array}\right)(t-1)(p-i+2)$. Since $\psi(H) \subseteq \partial^{i-1} G^{k}$, the 
induction hypothesis gives $|\psi(H)| \leq\left|\partial^{i-1} G^{k}\right|=O\left(n^{t-2}\right)$. Consequently $|H|=O\left(n^{t-2}\right)$.

\subsection{Proofs for $p \in\{t, t+1\}$ and $q=t-2$}

We need a proposition, which can be considered as an extension of Proposition 3.1.

Proposition 3.6. Let $q \leq p^{\prime} \leq p$, and $m=\left(\begin{array}{l}t \\ q\end{array}\right)(p-q)+1$. Suppose that $G$ is a p-graph on $[n]$ and $\phi$ is a function from $G$ to $\left(\begin{array}{c}{[n]} \\ p^{\prime}\end{array}\right)$ such that $\phi(E) \subseteq E$ for each $E \in G$. If $G \nrightarrow K_{t}^{q}$, then $\phi(G) \nrightarrow K_{t}^{q}(m)$.

Proof. Suppose instead, that $\phi(G) \rightarrow K_{t}^{q}(m)$. Then there are disjoint vertex sets $X_{1}, X_{2}, \ldots, X_{t}$ of size $m$ such that the following holds. Let $\mathcal{Q}$ be the family of $q$-sets having non-empty intersection with exactly $q$ of $X_{1}, X_{2}, \ldots, X_{t}$. For each $Q \in \mathcal{Q}$, there exists $E \in G$ such that $Q \subseteq \phi(E) \subseteq E$. Denote such $E$ by $E_{Q}$. We say that a set $Q \in \mathcal{Q}$ is bad if there exists $j$ such that $Q \cap X_{j}=\emptyset$ and $\left(E_{Q} \backslash Q\right) \cap X_{j} \neq \emptyset$. Given a bad $Q \in \mathcal{Q}$, a $t$-tuple $x_{1}, \ldots, x_{t}$ with $x_{i} \in X_{i}$ is called bad because of $Q$ if $\left\{x_{1}, \ldots, x_{t}\right\}$ contains $Q$ and at least one vertex from $E_{Q} \backslash Q$. A $t$-tuple from $X_{1} \times \cdots \times X_{t}$ is called bad if it is bad because of some $Q$. For fixed bad $Q \in \mathcal{Q}$, the number of bad $t$-tuples because of $Q$ is at most $(p-q) m^{t-q-1}$ (first select a vertex from $E_{Q} \backslash Q$ and then decide the remaining $t-q-1$ coordinates). The total number of bad $t$-tuples is thus at most $\left(\begin{array}{l}t \\ q\end{array}\right) m^{q}(p-q) m^{t-q-1}$. When $m>\left(\begin{array}{l}t \\ q\end{array}\right)(p-q)$, we have $\left(\begin{array}{l}t \\ q\end{array}\right) m^{q}(p-q) m^{t-q-1}<m^{t}$, or the number of bad $t$-tuples is less than the total number of $t$-tuples in $X_{1} \times \cdots \times X_{t}$. Hence there always exists a good $t$-tuple $T$ and consequently $\left.G\right|_{T} \supseteq K_{t}^{q}$, a contradiction.

Proof of (10) for $p=t$. Given $G \subseteq\left(\begin{array}{c}{[n]} \\ t\end{array}\right)$ such that $G \nrightarrow K_{t}^{q}$, we partition $G$ into $G_{t}+\ldots+G_{1}$ as in the beginning of Section 3.2. By Lemma 3.3, $\left|G^{t}\right|=O\left(n^{t-2}\right)$ and consequently $\left|G_{t}\right| \leq\left|G^{t}\right|=O\left(n^{t-2}\right)$. Trivially $\left|G_{i}\right| \leq\left|G^{i}\right|=O\left(n^{t-2}\right)$ for $i \leq t-2$. It remains to show that $\left|G_{t-1}\right| \leq L^{t-1}\left(n, K_{t}^{q}\right)+o\left(n^{t-1}\right)$. In fact, for each $E \in G_{t-1}$, we define $\phi(E)=D$ where $D$ is one of the $(t-1)$-subsets of $E$ satisfying $\operatorname{deg}(D)=1$. Proposition 3.6 implies that $\phi(G) \nrightarrow K_{t}^{q}(m)$ for $m=\left(\begin{array}{l}t \\ q\end{array}\right)(p-q)+1$. So

$$
|G|=|\phi(G)| \leq L^{t-1}\left(n, K_{t}^{q}(m)\right) \leq L^{t-1}\left(n, K_{t}^{q}\right)+o\left(n^{t-1}\right),
$$

where the last inequality follows from Lemma 2.4.

Proof of (10) for $p=t+1$. We need Lovász's version [11] of the Kruskal-Katona Theorem: let $H$ be $a(t+1)$-graph with $|H|=\left(\begin{array}{c}x \\ t+1\end{array}\right)$ for some real number $x$. Then $\partial H \geq\left(\begin{array}{l}x \\ t\end{array}\right)$. This 
implies that if $|\partial H|=O\left(n^{k}\right)$, then $|H|=O\left(n^{\frac{k(t+1)}{t}}\right)$. To see this, suppose that $|\partial H| \leq C n^{k}$ for some $C>0$. Since $\left(\frac{x}{t}\right)^{t} \leq\left(\begin{array}{l}x \\ t\end{array}\right) \leq|\partial H| \leq C n^{k}$, we have $\frac{x}{t} \leq C^{\frac{1}{t}} n^{\frac{k}{t}}$ and

$$
|H|=\left(\begin{array}{c}
x \\
t+1
\end{array}\right)=\left(\begin{array}{l}
x \\
t
\end{array}\right) \frac{x-t}{t+1} \leq C n^{k} C^{\frac{1}{t}} n^{\frac{k}{t}}=O\left(n^{\frac{k(t+1)}{t}}\right) .
$$

Now given $G \subseteq\left(\begin{array}{c}{[n]} \\ t+1\end{array}\right)$ such that $G \nrightarrow K_{t}^{q}$, we partition $G$ into $G_{t+1}+G_{t}+\ldots+G_{1}$. The proof of the $p=t$ case shows $\sum_{i=1}^{t}\left|G_{i}\right| \leq L^{t-1}\left(n, K_{t}^{q}\right)+o\left(n^{t-1}\right)$. It suffices to show that $\left|G_{t+1}\right|=o\left(n^{t-1}\right)$, or $\left|G^{t+1}\right|=o\left(n^{t-1}\right)$. Lemma 3.3 guarantees that $\partial^{t}\left(G^{t+1}\right)=O\left(n^{t-2}\right)$ and consequently, by the result of Lovász, $\left|G^{t+1}\right|=O\left(n^{\frac{(t-2)(t+1)}{t}}\right)=o\left(n^{t-1}\right)$.

Proof of (10) for $q=t-2$. Given $G \subseteq\left(\begin{array}{c}{[n]} \\ p\end{array}\right)$ such that $G \nrightarrow K_{t}^{t-2}$, we partition $G$ into $G_{p}+\ldots+G_{t}+G_{t-1}+\ldots+G_{1}$. The proof of the $p=t$ case shows $\sum_{i=1}^{t}\left|G_{i}\right| \leq$ $L^{t-1}\left(n, K_{t}^{q}\right)+o\left(n^{t-1}\right)$. For $t<k \leq p$, we apply Lemma 3.4 with $i=k$ and obtain that $\left|G_{k}\right| \leq\left|G^{k}\right| \leq \partial^{k}\left(G^{k}\right)=O\left(n^{t-2}\right)$, thus completing the proof.

\section{An Exact Result}

In order to prove Theorem 1.7, we need the following lemma, which can be proved by following the original proof of Mantel's Theorem [12]. We use + instead of $\cup$ for a disjoint union. In a graph $G$, given a vertex set $A$ and a vertex $x, N(x, A)$ denotes the neighborhood of $x$ in $A$, and $d(x, A)=|N(x, A)|$, in particular $d(x)=d(x, V(G))$. For disjoint vertex sets $X$ and $Y$, we denote by $e(X, Y)$ the number of edges between $X$ and $Y$. For simplicity we write $a b$ instead of $\{a, b\}$.

Lemma 4.1. Let $G=(V, E)$ be a triangle-free graph such that

for every $a b \in E$, there exists $c \in V$, such that $a c \notin E$ and $b c \notin E$.

Then $|E| \leq\left\lfloor\frac{(n-1)^{2}}{4}\right\rfloor+1$ with equality only when $G$ has the following structure: $V(G)=$ $A+B+\{z\}$, there exist $a \in A$ and a non-empty set $B_{z} \subseteq B$ such that $E(G)=A \times B-\{a b$ : $\left.b \in B_{z}\right\}+\left\{z b: b \in B_{z}\right\}+\{a z\}$.

Proof. Let $x y$ be an edge. Since $G$ is triangle-free, we have $N(x) \cap N(y)=\emptyset$. With $(\star)$, we further derive that $d(x)+d(y) \leq n-1$. 
If $d(x)+d(y) \leq n-2$ for every edge $x y$ in $G$, then following Mantel's proof of his theorem, we have

$$
\begin{gathered}
\frac{4|E|^{2}}{n}=\frac{\left(\sum_{x \in V} d(x)\right)^{2}}{n} \leq \sum_{x \in V}(d(x))^{2}=\sum_{x y \in E}(d(x)+d(y)) \leq(n-2)|E|, \\
|E| \leq \frac{n(n-2)}{4}<\frac{(n-1)^{2}}{4}<\left\lfloor\frac{(n-1)^{2}}{4}\right\rfloor+1 .
\end{gathered}
$$

Otherwise assume that $d(x)+d(y)=n-1$ for some $e=\{x, y\}$. Let $A=N(y)$ and $B=N(x)$. We know that $A \cap B=\emptyset$ and $A \cup B=V-\{z\}$ for some vertex $z$. Let $d_{1}=d(z, A)$ and $d_{2}=d(z, B)$.

Case 1: $d_{1}=0$, or $d_{2}=0$.

Say, $d_{1}=0$. For each $b \in N(z, B)$, there exists $a \in A$ such that $a b \notin E$, since otherwise edge $x b$ does not satisfy $(\star)$. This implies that

$$
|E|=e(A, B)+d(z, B) \leq|A||B| \leq\left\lfloor\frac{(n-1)^{2}}{4}\right\rfloor .
$$

Case 2: $d_{1}, d_{2}>0$.

In this case $d_{1} d_{2}-d_{1}-d_{2}+1=\left(d_{1}-1\right)\left(d_{2}-1\right) \geq 0$ with equality if and only if at least one of $d_{1}, d_{2}$ is 1 . Since $G$ is triangle-free, there is no edge between $N(z, A)$ and $N(z, B)$. Thus $e(A, B) \leq|A||B|-d_{1} d_{2}$ and

$$
|E|=e(A, B)+d(z, A)+d(z, B) \leq|A||B|-d_{1} d_{2}+d_{1}+d_{2} \leq\left\lfloor\frac{(n-1)^{2}}{4}\right\rfloor+1,
$$

where equality holds only when $G$ has the desired structure.

Proof of Theorem 1.7. To show that $L^{3}\left(n, K_{3}\right) \geq\left\lfloor\frac{(n-1)^{2}}{4}\right\rfloor$, we enlarge each edge of $K_{\left\lfloor\frac{n-1}{2}\right\rfloor,\left\lceil\frac{n-1}{2}\right\rceil}$ with the same new vertex.

To prove the upper bound, we consider a 3-graph $H$ on $[n]$ such that $H \nrightarrow K_{3}$. The proof of the $q=t-1$ case of Theorem 1.4 implies that each triple $T \in H$ contains a pair $\phi(T)$ with $\operatorname{deg}_{H}(\phi(T))=1$. We thus obtain a graph $G$ on $[n]$ with edge set $E=\{\phi(T): T \in H\}$. Clearly $|E|=|H|$, and $G$ satisfies ( $\star$ ) because

$$
\text { if } \phi(\{a, b, c\})=a b \text {, then } a c \notin E \text { and } b c \notin E \text {. }
$$


Next we claim that $G \neq G *$, where $G *$ is a graph causing the equality in Lemma 4.1. Suppose, to the contrary, that $G=G *$. Let us consider edges $z a$ and $z b$ for any $b \in B_{z}$. By (11), $\phi^{-1}(z a)=\{z, a, x\}$ for some $x \in A \backslash\{a\}$, and $\phi^{-1}(z b)=\{z, b, y\}$ for some $y \in B \backslash B_{z}$. Since $a$ is the unique vertex which is non-adjacent to both $x$ and $b$, we have $\phi^{-1}(x b)=$ $\{a, b, x\}$. The trace of $\{z, a, x\},\{z, b, y\},\{a, b, x\}$ on $\{z, a, b\}$ is a $K_{3}$, contradicting $H \nrightarrow K_{3}$.

Finally we apply Lemma 4.1 and obtain that $|H|=|E| \leq\left\lfloor\frac{(n-1)^{2}}{4}\right\rfloor$.

\section{Concluding Remarks and Open Problems}

A less ambitious goal than proving Conjecture 1.6 is to verify (3), or equivalently (10), for $p \geq t+2$ and $q \leq t-3$. This will reduce the trace problem to determining $\operatorname{ex}\left(n, H_{q, t}^{t-1}\right)$, which is only known for $q=2$. To obtain the asymptotic value of $L^{p}\left(n, K_{t}^{q}\right)$ in other cases, one should try to verify (6) for $p \geq t+2$ and $t \geq 5$; the smallest open case is to prove that

$$
L^{7}\left(n, K_{5}\right)=\left|T_{4}^{4}(n)\right|+o\left(n^{4}\right)=\left(\frac{n}{4}\right)^{4}+o\left(n^{4}\right) .
$$

Following the ideas in Sections 3.2 and 3.3, in order to extend Theorem 1.4 for all $p \geq t$, one needs to show that $G^{k}=o\left(n^{t-1}\right)$ for $t \leq k \leq p$. When $p \geq t+2$, this does not follow from Lemma 3.3 and the Kruskal-Katona theorem. The proof of Lemma 3.4 relies on the assumption $q=t-2$, and does not seem to generalize to other values of $q$.

A general uniform trace problem is to determine $L^{p}(n, F)$ for arbitrary $p$ and $F$. Because of the close connection between trace problems and Turán problems, as seen in Proposition 1.3 and Theorem 1.4, it is very hard to determine $L^{p}(n, F)$ in general. Let us consider $L^{3}(n, F)$ when $F$ is a graph. Fix $t=\chi(F)$. When $t \geq 4$, we have

$$
L^{3}(n, F)=\left|T_{t-1}^{3}(n)\right|+o\left(n^{3}\right)=\left(\begin{array}{c}
t-1 \\
3
\end{array}\right)\left(\frac{n}{t-1}\right)^{3}+o\left(n^{3}\right) .
$$

In fact, the lower bound for $L^{3}(n, F)$ follows from $T_{t-1}^{3}(n) \not \rightarrow F$, where $T_{t-1}^{3}(n)$ is the generalized Turán graph defined in the introduction. The reason for $T_{t-1}^{3}(n) \nrightarrow F$ is that when embedding $F$ into a $(t-1)$-partite graph, some partition set must contain both ends of an edge of $F$. The upper bound follows from (5) and Lemma 2.4. The same arguments actually show that $L^{p}(n, F)=\left|T_{t-1}^{p}(n)\right|+o\left(n^{t-1}\right)$ for every $F$ with $t=\chi(F)>p$.

Problem 5.1. Determine the order of magnitude of $L^{3}(n, F)$ for every $F$ with $\chi(F) \leq 3$. 
This seems no easier than determining the order of magnitude of the Turán numbers for bipartite graphs. We can derive an upper bound for $L^{3}(n, F)$ as follows. A result of Erdös

[2] implies that $\operatorname{ex}\left(n, K_{3}^{3}(m)\right)=O\left(n^{3-\frac{1}{m^{2}}}\right)$. For a 3-graph $H$, it is clear that $K_{3}^{3}(m) \subseteq H$ implies that $H \rightarrow K_{3}(m-1)$. For each $F$ with $\chi(F) \leq 3$, there exists $m$ such that $F \subseteq K_{3}(m)$. Hence $L^{3}(n, F) \leq L^{3}\left(n, K_{3}(m)\right) \leq \operatorname{ex}\left(n, K_{3}^{3}(m+1)\right)=O\left(n^{3-c}\right)$, where $c=1 /(m+1)^{2}$. However, we do not have a matching lower bound. For example, we only know $L^{3}\left(n, K_{3}(2)\right)=\Omega\left(n^{5 / 2}\right)$, in contrast to the upper bound $O\left(n^{26 / 9}\right)$ derived by above arguments (or $O\left(n^{11 / 4}\right)$ by some extra ideas). This lower bound can be seen from the 3partite 3-graph with partition sets $A, B, C$ of size $n$, and the edge set $\{e \cup v: v \in C, e \in G\}$, where $G$ is a maximum $C_{4}$-free bipartite graph on $(A, B)$ with $\Omega\left(n^{3 / 2}\right)$ edges.

\section{References}

[1] J. Balogh, P. Keevash and B. Sudakov, Disjoint representability of sets and their complements, J. Combin. Theory Ser. B 95 (2005), no. 1, 12-28.

[2] P. Erdős, On extremal problems of graphs and generalized graphs, Israel J. Math. 2 (1964), 183-190.

[3] P. Erdös, R. Rado, Intersection theorems for systems of sets, J. London Math. Soc. 35 (1960), 85-90.

[4] P. Erdős, Chao Ko, R. Rado, Intersection theorems for systems of finite sets. Quart. J. Math. Oxford Ser. (2) 12 (1961), 313-320.

[5] P. Erdős, M. Simonovits, Supersaturated graphs and hypergraphs. Combinatorica 3 (1983), no. 2, 181-192.

[6] P. Frankl, J. Pach, On disjointly representable sets, Combinatorica 4 (1984), 39-45.

[7] Z. Füredi, On finite system whose every intersection is a kernal for a star, Discrete Math. 47 (1983), 129-132.

[8] Z. Füredi, Turán type problems. Surveys in combinatorics, 1991 (Guildford, 1991), 253300, London Math. Soc. Lecture Note Ser., 166, Cambridge Univ. Press, Cambridge, 1991. 
[9] Z. Füredi, J. Pach, Traces of finite sets: extremal problems and geometric applications. Extremal problems for finite sets (Visegrád, 1991), 251-282, Bolyai Soc. Math. Stud., 3, János Bolyai Math. Soc., Budapest, 1994.

[10] Z. Füredi, F. Quinn, Traces of finite sets. Ars Combin. 18 (1984), 195-200.

[11] L. Lovász, Combinatorial problems and exercises, North-Holland, Amsterdam, 1979.

[12] W. Mantel, Problem 28, Wiskundige Opgaven 10 (1907), 60-61.

[13] D Mubayi, A hypergraph extension of Turan's theorem, J. Combin. Theory Ser. B 96 (2006), no. 1, 122-134.

[14] O. Pikhurko, Exact Computation of the Hypergraph Turan Function for Expanded Complete 2-Graphs, J. Combin. Theory Ser. B, accepted.

[15] N. Sauer, On the density of families of sets, J. Combinatorial Theory Ser. A 13 (1972), $145-147$.

[16] S. Shelah, A combinatorial problem; stability and order for models and theories in infinitary languages, Pacifc J. Math. 41 (1972), 247-261.

[17] P. Turán, On an extremal problem in graph theory. Mat. Fiz. Lapok 48 (1941), 436-452.

[18] V. N. Vapnik and A. Ya. Chervonenkis, On the uniform convergence of relative frequencies of events to their probabilities, Theory Probab. Appl. 16 (1971), 264-280. 